\title{
REDUCIENDO LAS INEQUIDADES EN SALUD Y MEJORANDO LA SALUD MATERNA MEDIANTE LA MEJORA DE LOS SISTEMAS DE INFORMACIÓN EN SALUD: WAWARED PERÚ
}

\author{
José E. Pérez-Lu1,a, Ruth Iguiñiz Romero ${ }^{1, b}$, Angela M. Bayer ${ }^{1, c}$, Patricia J. García ${ }^{1, d}$ \\ RESUMEN \\ En los países en desarrollo no existen datos de calidad para apoyar la toma de decisiones y la gobernanza, debido a \\ inadecuados procesos de colección y transmisión de información. Nuestro proyecto WawaRed-Perú: "Reduciendo las \\ inequidades en salud y mejorando la salud materna mediante la mejora de los sistemas de información en salud" propone \\ mejorar los procesos e indicadores de salud materna a través de la implementación de estándares de interoperabilidad \\ en los sistemas de información de salud materna para que los tomadores de decisión tengan información oportuna y \\ de calidad. Con esto se desea apoyar a desarrollar mejores políticas de salud y, a su vez, contribuir a disminuir los \\ problemas de equidad en salud de las mujeres peruanas, y potencialmente las mujeres de otros países en desarrollo. \\ El objetivo de este artículo es presentar la situación actual de los sistemas de información de salud materna en el Perú. \\ Palabras clave: Sistemas de Información en Salud; Servicios de salud materna; Registros médicos; Embarazo; \\ Informática médica (fuente: DeCS BIREME).

\section{WAWARED PERU: REDUCING HEALTH INEQUITIES AND IMPROVING MATERNAL HEALTH BY IMPROVING INFORMATION SYSTEMS IN HEALTH}

\begin{abstract}
In developing countries, there are no high quality data to support decision-making and governance due to inadequate information collection and transmission processes. Our project WawaRed-Peru: "Reducing health inequities and improving maternal health by improving health information systems" aims to improve maternal health processes and indicators through the implementation of interoperability standards for maternal health information systems in order for decision makers to have timely, high quality information. Through this project, we hope to support the development of better health policies and to also contribute to reducing problems of health equity among Peruvian women and potentially women in other developing countries. The aim of this article is to present the current state of information systems for maternal health in Peru.
\end{abstract}

Key words: Health Information Systems; Maternal health services; Medical records; Pregnancy; Medical informatics (source: MeSH NLM).

\section{INTRODUCCIÓN}

La salud materna es una prioridad para el Perú y el mundo desde la década de los 80 promovida por la "Iniciativa por Maternidad Segura" ${ }^{(1,2)}$, la Conferencia de Población y Desarrollo en El Cairo ${ }^{(3)}$ y, más recientemente, los Objetivos de Desarrollo del Milenio ${ }^{(4,5)}$. El Perú ha reducido su tasa de mortalidad materna de 234,5 por 100000 nacidos vivos en 1990 a 96 por 100000 nacidos vivos en 2010, estando muy cerca de lograr el objetivo del milenio número 5 , disminuir la mortalidad materna en el $2015^{(6,7)}$.

Está demostrado que es posible mejorar la salud de la madre y del recién nacido con acciones preventivas y cuidados obstétricos de emergencia, para prevenir y resolver complicaciones durante el embarazo y el parto ${ }^{(8-12)}$. Sin embargo, la implementación de esas acciones por parte de los prestadores de salud y los tomadores de decisiones necesitan buenos sistemas de información de salud que guíen sus decisiones ${ }^{(13)}$.

\footnotetext{
Facultad de Salud Pública y Administración, Universidad Peruana Cayetano Heredia. Lima, Perú.

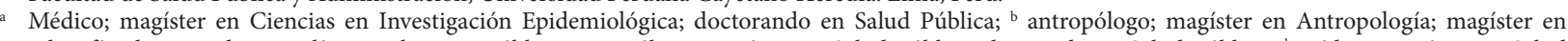
Filosofía; doctorando en políticas urbanas y públicas; ${ }^{c}$ socióloga; magíster en Salud Pública; doctorado en Salud Pública; ${ }^{\text {d }}$ médico, magíster en Salud Pública; doctor en Medicina; especialista en enfermedades infecciosas

Recibido: : 03-04-15 Aprobado: 13-05-15
}

Citar como: Pérez-Lu JE, Iguiñiz Romero R, Bayer AM, García PJ. Reduciendo las inequidades en salud y mejorando la salud materna mediante la mejora de los sistemas de información en salud: Wawared Perú. Rev Peru Med Exp Salud Publica. 2015;32(2):373-7. 


\section{IMPORTANCIA DE LOS SISTEMAS DE INFORMACIÓN PARA MEJORAR LA TOMA DE DECISIONES EN SALUD MATERNA}

Los sistemas de salud, las estructuras y los procesos involucrados en la prestación de servicios de salud requieren lideres informados ${ }^{(14)}$. Desde esta perspectiva, se necesitan sistemas de información robustos y efectivos, para lo cual se requieren procesos y personas capaces de garantizar la colección, procesamiento, análisis y distribución de datos de manera consistente, exacta y oportuna, para la toma de decisiones ${ }^{(15,16)}$.

Desafortunadamente, el personal de salud no siempre está capacitado en la colección o análisis de datos o informática. Por lo tanto, la calidad de datos variaba mucho entre regiones de zonas rurales versus urbanas $(17,18)$ y entre bases de datos.

\section{ANTECEDENTES: PROYECTO WAWARED}

En 2010 implementamos el proyecto WawaRed "Conectándose para una mejor salud materno-infantil en el Perú" con financiamiento del Programa Mobile Citizen del Banco Interamericano de Desarrollo (BID) (http:// wawared.org/). WawaRed se implementó en el hospital de Ventanilla y los 15 centros de salud de Ventanilla, región de Callao, con una población de 300000 habitantes ${ }^{(19)}$.

El proyecto consistió en la implementación de 1). Una historia clínica electrónica (HCE) para la atención prenatal que reduce el tiempo de llenado de formatos; 2). Envío de mensajes de texto (SMS) personalizados a las mujeres embarazadas, y 3). Un sistema de respuesta de voz interactiva (IVR). Los SMS envían información a la gestante referente a su embarazo. EI IVR es una central telefónica con mensajes de voz pregrabados con información sobre los signos alarma.

El sistema tenido gran éxito y aceptación, pero el personal de salud todavía debe remitir los formularios impresos (que ahora puede digitar una vez e imprimir) a los diferentes niveles del sistema de salud hasta que llegan al Ministerio de Salud.

\section{MARCO CONCEPTUAL Y LOS OBJETIVOS DE UN NUEVO PROYECTO: WAWARED PERÚ}

La experiencia de implementación de la HCE trajo a la luz el problema de la falta de integración e interacción de los diferentes sistemas de información de salud materna, y la necesidad de garantizar la calidad de información a lo largo de su recorrido por los distintos niveles del sistema de salud.

Así, se plantea este nuevo proyecto WawaRed Perú, que busca desarrollar una herramienta para que los diferentes sistemas de información de salud sean interactivos e interoperables. Se define interoperabilidad como la habilidad de intercambiar y utilizar información entre diferentes sistemas. También incluye un componente de capacitación, para entrenar al personal responsable de la colección y manejo de datos en el procesamiento y análisis de la información para la toma de decisiones, en el nivel local e intermedio del sistema de salud.

La Figura 1 ilustra el marco conceptual; en él se muestran las necesidades, productos y las actividades propuestas en el proyecto. Las necesidades se enfocan en tener la información adecuada, y a tiempo, para que sea analizada por tomadores de decisiones calificados. Para ello integraremos los sistemas de información y capacitaremos a las personas claves. Con esto esperamos tener información a tiempo y de mayor calidad, y personal entrenado para tomar mejores decisiones que pueden mejorar la gobernanza, disminuir las inequidades y, como resultado final, la morbimortalidad materna.

Este artículo tuvo el objetivo de presentar los hallazgos iniciales del nuevo proyecto WawaRed con relación a los problemas relacionados a los sistemas de información de salud materna en el país.

\section{PROBLEMAS EN LOS SISTEMAS DE INFORMACIÓN DE SALUD MATERNA EN PERÚ}

El proyecto WawaRed-Perú se inició realizando una investigación formativa, con el objetivo de conocer la situación actual de los sistemas de información de salud materna y las necesidades del personal de salud en Lima, Callao y Ayacucho. Se realizaron cuatro grupos focales con personal de salud de centros de salud de primer nivel, centros de salud maternos y hospitales encargados de la atención de las gestantes. También se realizaron once entrevistas a profundidad a personas clave de las estrategias de salud sexual y reproductiva, de la oficina de estadística e informática y la oficina de epidemiología de las direcciones regionales de salud del Callao, Ayacucho, Lima Este, Lima Norte, Lima Sur y Lima Provincia. Estas actividades se realizaron en el periodo de noviembre de 2013 a enero de 2014. 


\section{Marco Conceptual}

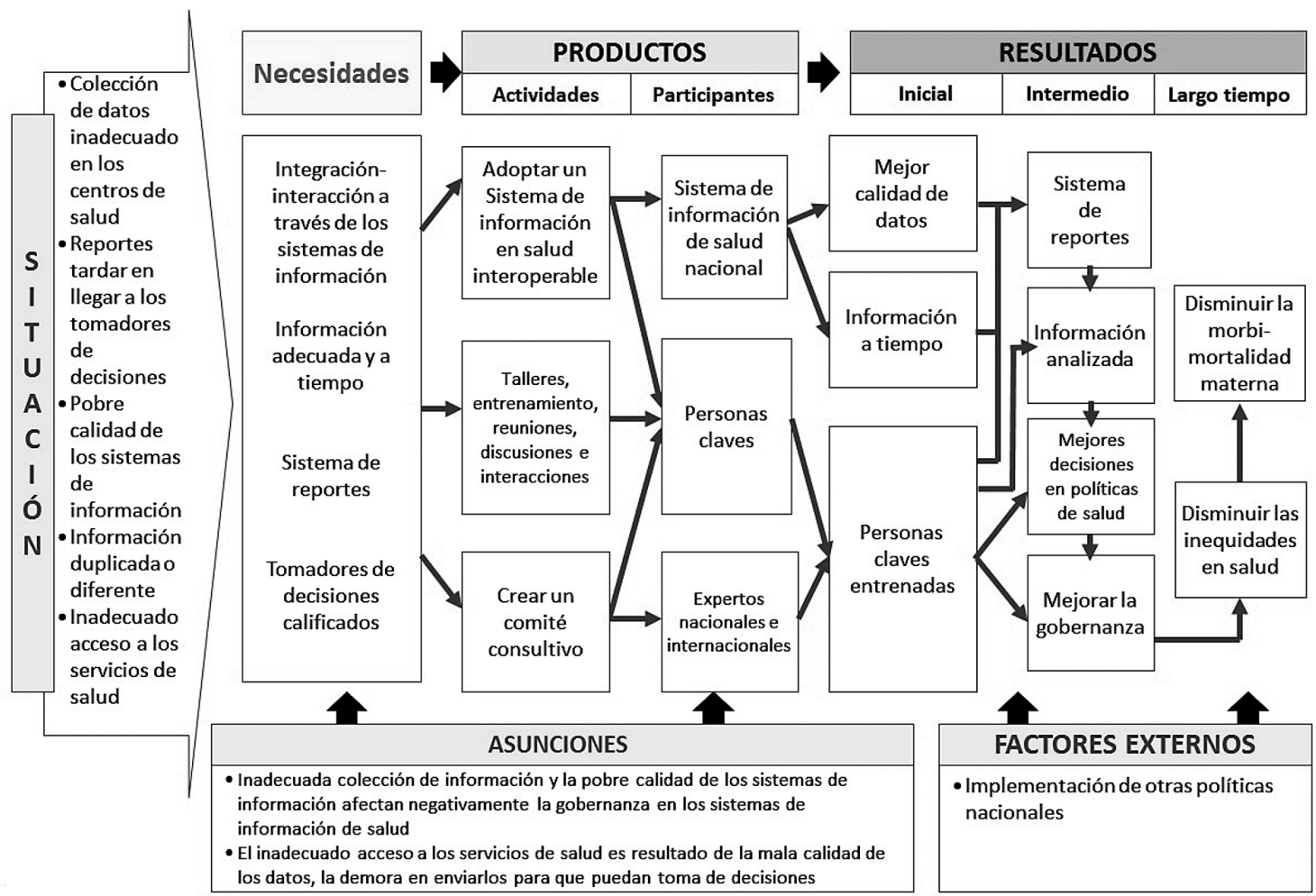

Figura 1. Marco conceptual para WawaRed Perú

La información cualitativa recogida nos permitió comprender mejor cuatro problemas relevantes para nuestro proyecto y relacionados a los sistemas de información en salud materna en el país.

Primero, los flujos de colección, procesamiento y análisis de la información reflejan un proceso unidireccional (Figura 2). El proceso se inicia cuando los datos son colectados por el proveedor de salud en la historia clínica, en el nivel de prestación de servicio. Luego, continúan su trayecto hacia los niveles intermedios de gestión y planificación del sector, como las microredes y redes, y después al nivel regional (por ej., DIRESA) y nacional (Ministerio de Salud) ${ }^{(20)}$. Una vez que la información llega a las instancias de decisión nacional, no existe un proceso eficiente y bien definido por el cual la información regresa a los niveles de decisión regional, intermedia y local. El nivel local de atención primaria también necesita esta información para que el prestador de salud pueda tomar decisiones de tipo asistencial basado en información oportuna, pero esta información nunca regresa ${ }^{(17)}$.

Al tener acceso limitado a los datos, el proveedor de salud a nivel local cuenta con pocos recursos y oportunidades para usar o analizar la información a nivel de su establecimiento de salud o de la comunidad que atiende en su conjunto. Por ejemplo, el proveedor de salud tiene acceso a la información de cada historia clínica en el contexto de la visita de una usuaria. Sin embargo, no tiene información consolidada oportuna acerca de las características colectivas o la problemática de salud colectiva de las gestantes de su comunidad, para adaptar la información que brinda a las gestantes a sus necesidades específicas.

\begin{tabular}{|c|c|c|c|c|c|}
\hline $\begin{array}{l}\text { Escrito en papel } \\
\text { en el } \\
\text { establecimiento } \\
\text { de salud }\end{array}$ & $\begin{array}{c}\text { Digitalizado en } \\
\text { el sistema en el } \\
\text { establecimiento } \\
\text { de salud }\end{array}$ & $\begin{array}{l}\text { Enviado a la } \\
\text { microred y se } \\
\text { vuelve a } \\
\text { digitalizar }\end{array}$ & $\begin{array}{c}\text { Enviado a la red } \\
\text { y se vuelve a } \\
\text { digitalizar }\end{array}$ & $\begin{array}{l}\text { Enviado a la } \\
\text { región y se } \\
\text { vuelve a } \\
\text { digitalizar }\end{array}$ & $\begin{array}{l}\text { Enviado a la } \\
\text { sede central del } \\
\text { Ministerio de } \\
\text { Salud }\end{array}$ \\
\hline
\end{tabular}

Figura 2. Flujo de la información desde el establecimiento de salud hasta el nivel central 
Segundo, la fragmentación de la atención y de los sistemas de información en salud materna en el Perú. Los servicios se brindan por especialidad y la información de las historias clínicas no se comparte apropiadamente entre servicios. Las historias clínicas de una usuaria en un nivel de atención primaria no están accesibles cuando la usuaria recurre a un establecimiento de salud más especializado. Por ejemplo, la atención de control prenatal y seguimiento a la gestante es brindada en su mayoría por obstetras en los centros de salud de primer nivel. Sin embargo, el parto ocurre en centros de mayor capacidad resolutiva. La usuaria lleva con ella un resumen de su historia clínica impresa (carnet de control prenatal), si la gestante lo pierde o se lo olvida, se pierde la información de sus controles prenatales previos y le tienen que realizar nuevos exámenes de laboratorio. Luego del parto, la información no es transferida al centro de salud de donde proviene la usuaria y que es responsable de la atención del puerperio y el control del niño (Figura 3). Esto ocasiona que el centro de salud no sepa del resultado del parto, y no podrá localizarla para sus controles de puerperio y el control de niño sano al recién nacido.

Tercero, el uso de las tecnologías de información y comunicación (TIC) puede verse limitado en algunas zonas donde los servicios de comunicaciones no son estables ni tan extendidos. Los establecimientos de salud que se encuentran en un área periurbana usualmente (no siempre), tienen alguna facilidad para acceder a servicios de TIC (ejm computadoras, conexión a Internet, etc.). Pero esto no ocurre en zonas rurales, que tiene muchas limitaciones para acceder a estas tecnologías.

Cuarto, la capacidad en el manejo de los datos por parte del personal de estadística en los establecimientos a nivel local, intermedio y nivel central es limitada. En la microrred, los datos son consolidados y elevados al nivel superior con fines de monitoreo, gestión y planificación de los servicios

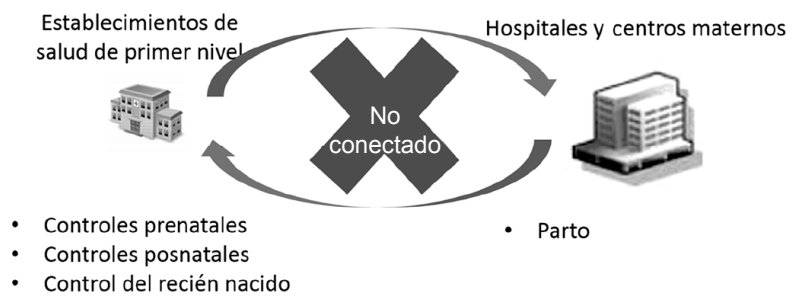

Figura 3. Flujo de información entre los establecimientos de salud de primer nivel y los hospitales y centros maternos. y de impacto en los indicadores de salud. Si bien el proveedor de salud del nivel local está capacitado para identificar los diagnósticos que reporta a nivel individual; el personal de estadística encargado de consolidar la información, redigitarla y transferirla hacia los niveles de gestión, en su mayoría no cuenta con conocimientos suficientes para entender la información de la historia clínica o su relevancia para la atención directa y la salud de las usuarias, o para realizar consolidados o informes relevantes para la institución.

\section{CONCLUSIONES}

Los dos primeros problemas encontrados, en relación al flujo de información y la fragmentación de los sistemas de información, están estrechamente ligados. El primer problema evidencia la necesidad de interconectar los procesos verticales de transmisión de información y lograr interconectividad en las dos direcciones: desde el nivel local hasta el central y viceversa. El segundo, evidencia la necesidad de interconectar procesos horizontales vinculados con la prestación del servicio: centro de salud-hospital-centro de salud. Para resolver estos problemas el proyecto está desarrollando un modelo de integración-interacción de información de la salud materna que permita: 1). El intercambio de información de la historia clínica electrónica (HCE) originada en el centro de salud, para el control prenatal, con una HCE hospitalaria, para el parto, y que regrese al centro para el control posparto y del niño sano; 2). La transmisión de información desde el nivel local hasta los niveles intermedios (redes, microrredes y las DIRESA) y central de toma de decisiones (MINSA) en tiempo real y confiable.

Los dos siguientes problemas están vinculados con las capacidades de infraestructura tecnológica y profesionales. Ante el tercer problema, en caso de ausencia de Internet constante en los establecimientos de salud que deseen utilizar la HCE, estamos desarrollando una aplicación que permite el recojo y almacenamiento de información en el sistema para un envío posterior.

Finalmente, como parte del proyecto, se está brindando capacitación a los obstetras en el llenado de la HCE y en el análisis de datos, y al personal de estadística e informática en el manejo de los sistemas y en el análisis de los datos.

Contribuciones de autoría: los cuatro autores concibieron la idea de investigación, obtuvieron el financiamiento y desarrollan el proyecto. JEPL y RIR hicieron la recolección de los datos y realizaron el análisis de los datos. JEPL, RIR y AMB 
interpretaron los resultados. JEPL desarrolló la primera versión del manuscrito, a la cual RIR, AMB y PJF colaboraron activa y críticamente. Los cuatro autores aprobaron la versión del manuscrito enviada.
Conflictos de interés: ninguno por declarar. La institución financiadora no participó en ninguna fase del estudio.

Fuentes de financiamiento: este trabajo se llevó a cabo con la ayuda de una subvención del Centro Internacional de Investigaciones para el Desarrollo, Ottawa, Canadá.

\section{REFERENCIAS BIBLIOGRÁFICAS}

1. Starrs AM. Safe motherhood initiative: 20 years and counting. Lancet. 2006 Sep 30;368(9542):1130-2.

2. International Conference on Safe Motherhood. Action Plan. Call to Action. Nairobi, Kenya; 1987.

3. McIntosh CA, Finkle JL. The Cairo conference on population and development: A new paradigm?. Popul Dev Rev. 1995;21(2):223-60.

4. United Nations. United Nations Millennium Declaration. New York: Resolution adopted by the General Assembly; 2000.

5. Torres C, Mújica O, Si $\backslash$ na E, Ho G, Qian H, MY K, et al. Salud, equidad y los objetivos de desarrollo del milenio. Rev Panam Salud Pública Pan. Rev Panam Salud Publica. 2004 Jun;15(6):430-9.

6. Instituto Nacional de Estadística e Informática. Encuesta demográfica y de salud familia 1996. Lima: INEI; 1997.

7. Watanabe T. Tendencia, Niveles y Estructura de la Mortalidad Materna en el Perú 1992-2000. Lima: INEI; 2002.

8. Miller S, Sloan N, Winikoff B, Langer A, Fikree FF. Where is the " $\mathrm{E}$ " in $\mathrm{MCH}$ ? The need for an evidencebased approach in safe motherhood. J Midwifery Women's Health. 2003 JanFeb;48(1):10-8.

9. Favin M, Bradford B, Cebula D. Improving maternal health in developing countries. Geneva: WFPHA; 1984.
10. Kerber KJ, de Graft-Johnson JE, Bhutta ZA, Okong P, Starrs A, Lawn JE. Continuum of care for maternal, newborn, and child health: from slogan to service delivery. Lancet. 2007 Oct 13;370(9595):1358-69.

11. Winikoff B. Women's health in developing countries. En: Wallace $H$, Gin K, eds. Health care of women and children in developing countries. Los Angeles: Third Party Publishers, 1990.

12. Rooney C. Antenatal care and maternal health: how effective is it? A review of the evidence. Geneva: WHO; 1992.

13. Ko H, Turner T, Jones C, Hill C. Patient-held medical records for patients with chronic disease: a systematic review. Qual Saf Health Care. 2010 Oct;19(5):e41. doi: 10.1136/qshc.2009.037531.

14. Beratarbide E, Kelsey T. eHealth governance, a key factor for better health care: Implementation of IT Governance to ensure better care through better eHealth. En: Brown $S$, Brown M. Ethical Issues and Security Monitoring Trends in Global Healthcare. Minneapolis: IGI Global Edinburgh; 2009.

15. World Health Organization. The World health report: 2000: Health systems: improving performance. Geneva: WHO; 2000.

16. Travis P, Bennett $S$, Haines A, Pang $T$, Bhutta Z, Hyder AA, et al. Overcoming health-systems constraints to achieve

$$
\begin{aligned}
& \text { the Millennium Development } \\
& \text { Goals. Lancet. } 2004 \text { Sep } \\
& \text { 4-10;364(9437):900-6. }
\end{aligned}
$$

17. Iguiñiz-Romero $\mathrm{R}$, Palomino $\mathrm{N}$. Data do count! Collection and use of maternal mortality data in Peru, 1990-2005, and improvements since 2005. Reprod Health Matters. 2012 Jun;20(39):174-84. doi: 10.1016/ S0968-8080(12)39605-5.

18. Almeida C. Reforma de sistemas de servicios de salud y equidad en América Latina y el Caribe: algunas lecciones de los años 80 y 90. Cad Saude Publica. 2002;18(4):905-25.

19. Curioso WH, Roman H, Perez-Lu J, Castagnetto JM, García PJ. Mejorando los sistemas de información en salud materna: validación de historias clínicas electrónicas en el Callao, Perú. Rev Peru Med Exp Salud Publica. 2010;27(3):487-9.

20. Curioso WH, Pardo K, Valeriano L. Uso de los establecimientos de salud del Ministerio de Salud del Perú, 20092011. Rev Peru Med Exp Salud Publica. 2013;30(2):175-80.

\footnotetext{
Correspondencia: José E Pérez-Lu

Dirección: Av. Honorio Delgado 430 - Urb.

Ingeniería-SMP.

Teléfono: +5114818283

Correo electrónico: jose.perez.l@upch.pe
} 